\title{
Stochastic Dominance under the Nonlinear Expected Utilities
}

\author{
Xinling Xiao ${ }^{1,2}$ \\ ${ }^{1}$ Shandong University Qilu Securities Institute for Financial Studies and School of Mathematics, Jinan 250100, China \\ ${ }^{2}$ School of Mathematical Sciences, Shandong Normal University, Jinan 250014, China \\ Correspondence should be addressed to Xinling Xiao; xinlingxiao@mail.sdu.edu.cn
}

Received 26 March 2014; Revised 28 May 2014; Accepted 19 June 2014; Published 31 August 2014

Academic Editor: Guangchen Wang

Copyright (C) 2014 Xinling Xiao. This is an open access article distributed under the Creative Commons Attribution License, which permits unrestricted use, distribution, and reproduction in any medium, provided the original work is properly cited.

\begin{abstract}
In 1947, von Neumann and Morgenstern introduced the well-known expected utility and the related axiomatic system (see von Neumann and Morgenstern (1953)). It is widely used in economics, for example, financial economics. But the well-known Allais paradox (see Allais (1979)) shows that the linear expected utility has some limitations sometimes. Because of this, Peng proposed a concept of nonlinear expected utility (see Peng (2005)). In this paper we propose a concept of stochastic dominance under the nonlinear expected utilities. We give sufficient conditions on which a random choice $X$ stochastically dominates a random choice $Y$ under the nonlinear expected utilities. We also provide sufficient conditions on which a random choice $X$ strictly stochastically dominates a random choice $Y$ under the sublinear expected utilities.
\end{abstract}

\section{Introduction}

In [1], von Neumann and Morgenstern introduced the wellknown expected utility and the related axiomatic system. It is widely used in economics, for example, financial economics. They exhibited four relatively modest axioms of "rationality" such that any agent satisfying the axioms has a utility function. They claimed that $U(\cdot)$ can be characterized by $U(X)=E[u(X)]$. That is to say they proved that an agent is (VNM-) rational if and only if there exists a real-valued function $u(\cdot)$ defined on possible outcomes such that every preference of the agent is characterized by maximizing the expected value of $u(\cdot)$, which can then be defined as the agent's VNM-utility. Here $u(\cdot): R \rightarrow$ $R$ is a continuous and strictly increasing function, and $E[\cdot]$ is the linear expectation in some probability space $(\Omega, \mathscr{F}, \mathbb{P})$.

However, some real world utilities cannot be represented by this expected utility. A famous counterexample is the wellknown Allais paradox (see [2]). Allais paradox shows that linear expected utility has some limitations sometimes.

In [3], Peng developed nonlinear expectation and sublinear expectation theory. G-expectation is a kind of special sublinear expectation. More details can be found in [4-7]. In [8-11], G-expectation is used in financial economics.

In [12], Peng developed a nonlinear type of von Neumann-Morgenstern representation theorem to utilities. He proved that there exists a nonlinear expected utility $U(\cdot)$, such that an agent $\hat{A}$ prefers a random choice $X$ than $Y$ which is formulated by $U(X)>U(Y)$.

But nonlinear expected utility can only describe an agent's preference; how to describe a group of agents' preference? In this paper we consider the question raised upward; to this end we define a corresponding concept of stochastic dominance under the nonlinear expected utilities.

The rest of this paper is organized as follows. In Section 2, we give some basic notions and results of nonlinear expectations and nonlinear expected utilities. In Section 3, we give the main results and the proofs.

\section{Nonlinear Expectations and Nonlinear Expected Utilities}

In this section we shall give some results of nonlinear expectations and nonlinear expected utilities. 
2.1. Nonlinear Expectations. We present some preliminaries in the theory of nonlinear expectations and sublinear expectations. The following definitions and properties can be found in [3].

Let $\Omega$ be a given set and let $\mathscr{H}$ be a linear space of real valued functions defined on $\Omega$ satisfying the following: if $X_{i} \in \mathscr{H}, i=1,2, \ldots, n$, then $\varphi\left(X_{1}, X_{2}, \ldots, X_{n}\right) \in \mathscr{H}$, for all $\varphi \in \mathscr{C}_{l \text {,lip }}(R)$, where $\mathscr{C}_{l \text {,lip }}(R)$ is the space of all real continuous functions defined on $R$ such that

$$
\begin{array}{r}
|\varphi(\mathbf{x})-\varphi(\mathbf{y})| \leq \mathscr{C}\left(1+\mathbf{x}^{k}+\mathbf{y}^{k}\right)|\mathbf{x}-\mathbf{y}| \\
\forall \mathbf{x}, \mathbf{y} \in R, k \text { depends on } \varphi
\end{array}
$$

Definition 1. $\mathbb{E}: \mathscr{H} \rightarrow R$ is said to be a nonlinear expectation defined on $\mathscr{H}$ if it satisfies the following.

(i) Monotonicity:

$$
\mathbb{E}(X) \geq \mathbb{E}(Y), \quad \text { if } X \geq Y .
$$

(ii) Constant Preserving:

$$
\mathbb{E}(c)=c, \quad \text { for } c \in R .
$$

A nonlinear expectation is called sublinear expectation if it also satisfies the following.

(iii) Subadditivity: for each $X, Y \in \mathscr{H}$,

$$
\mathbb{E}(X+Y) \leq \mathbb{E}(X)+\mathbb{E}(Y) .
$$

(iv) Positive homogeneity:

$$
\mathbb{E}(\lambda X)=\lambda \mathbb{E}(X), \quad \text { for } \lambda \geq 0 \text {. }
$$

The triple $(\Omega, \mathscr{H}, \mathbb{E})$ is called nonlinear expectation space and sublinear expectation space correspondingly.

Definition 2. Let $\mathbb{E}_{1}$ and $\mathbb{E}_{2}$ be two nonlinear expectations defined on $(\Omega, \mathscr{H})$; $\mathbb{E}_{1}$ is said to be dominated by $\mathbb{E}_{2}$ if

$$
\mathbb{E}_{1}(X)-\mathbb{E}_{1}(Y) \leq \mathbb{E}_{2}(X-Y), \quad \text { for } X, Y \in \mathscr{H}
$$

Remark 3. From (iii), a sublinear expectation is dominated by itself. In many situations, (iii) is also called the property of self-domination. It is easy to conclude that in a sublinear expectation space $(\Omega, \mathscr{H}, \mathbb{E}),-\mathbb{E}(-X) \leq \mathbb{E}(X)$, for $X \in \mathscr{H}$. If $-\mathbb{E}(-X)=\mathbb{E}(X)$, we say $X$ has no mean uncertainty.

Theorem 4 (Represent theorem). Let $\mathbb{E}$ be a functional defined on a linear space $\mathscr{H}$ satisfying subadditivity and positive homogeneity. Then there exists a family of linear functionals defined on $\mathscr{H}$ such that

$$
\mathbb{E}(X)=\sup _{\mathbb{P} \in \mathscr{P}} \mathbb{E}_{\mathbb{P}}(X), \quad \text { for } X \in \mathscr{H}
$$

and, for each $X \in \mathscr{H}$, there exists $\mathbb{P}_{X} \in \mathscr{P}$ such that $\mathbb{E}(X)=$ $\mathbb{E}_{\mathbb{P}_{X}}(X)$.

Furthermore, if $\mathbb{E}$ is a sublinear expectation, then the corresponding $\mathbb{E}_{\mathbb{P}_{X}}$ is a linear expectation.
According to the represent theorem, if $\mathbb{E}$ is a sublinear expectation, we have

$$
\mathbb{E}(X)=\sup _{\mathbb{P} \in \mathscr{P}} \mathbb{E}_{\mathbb{P}}(X), \quad \text { for } X \in \mathscr{H} .
$$

Suppose $(\Omega, \mathscr{F})$ is a measurable space, for such $\mathscr{P}$, we can define an upper probability

$$
\mathbb{V}(A)=\sup _{\mathbb{P} \in \mathscr{P}} \mathbb{P}(A), \quad A \in \mathscr{F}
$$

and a lower probability

$$
v(A)=\inf _{\mathbb{P} \in \mathscr{P}} \mathbb{P}(A), \quad A \in \mathscr{F} .
$$

Obviously $\mathbb{V}$ and $v$ are conjugated to each other; that is,

$$
\mathbb{V}(A)+v\left(A^{c}\right)=1
$$

where $A^{c}$ is the complementary set of $A$.

Definition 5. A set $A$ is polar if $\mathbb{V}(A)=0$. A property holds quasisurely (q.s). if it holds outside a polar set.

Definition 6. Let $X$ be a given random variable on a nonlinear expectation space $(\Omega, \mathscr{H}, \mathbb{E})$. One defines a functional on $\mathscr{C}_{l, \text { lip }}(R)$ by

$$
\mathbb{F}_{X}[\varphi]:=\mathbb{E}[\varphi(X)]: \varphi \in \mathscr{C}_{l, \text { lip }}(R) \longrightarrow R .
$$

$\mathbb{F}_{X}$ is called the distribution of $X$ under $\mathbb{E}$.

Definition 7. Let $X_{1}$ and $X_{2}$ be two random variables defined on nonlinear expectation spaces $\left(\Omega_{1}, \mathscr{H}_{1}, \mathbb{E}_{1}\right)$ and $\left(\Omega_{2}, \mathscr{H}_{2}, \mathbb{E}_{2}\right)$, respectively. They are called identically distributed, denoted by $X_{1} \stackrel{d}{=} X_{2}$, if

$$
\mathbb{E}_{1}\left[\varphi\left(X_{1}\right)\right]=\mathbb{E}_{2}\left[\varphi\left(X_{2}\right)\right], \quad \text { for } \varphi \in \mathscr{C}_{l, \text { lip }}(R) .
$$

It is clear that $X_{1} \stackrel{d}{=} X_{2}$ if and only if their distributions coincide. One says that the distribution of $X_{1}$ is stronger than that of $X_{2}$ if

$$
\mathbb{E}_{1}\left[\varphi\left(X_{1}\right)\right] \geq \mathbb{E}_{2}\left[\varphi\left(X_{2}\right)\right], \quad \text { for each } \varphi \in \mathscr{C}_{l, \text { lip }}(R) .
$$

2.2. Nonlinear Expected Utilities. The following definitions and properties can be found in [12]. Let $\mathbb{E}$ be a self-dominated nonlinear expectation defined on $\mathscr{H}$. Define a quasinorm $\|X\|_{* \infty}:=\inf _{\omega \in \Omega}\{c \in R ; c \geq|X|$ in $\mathscr{H}\}$. A utility functional of an agent $\hat{\mathrm{A}}$ is a real functional $U: \mathscr{H} \rightarrow R$. This functional satisfies the following obvious axioms:

(u1) monotonicity: if $X \geq Y$ in $\mathscr{H}$, then $\mathbb{U}(X) \geq \mathbb{U}(Y)$, and if $X \geq Y$ and $\|X-Y\|_{*}>0$, then $\mathbb{U}(X)>\mathbb{U}(Y)$;

(u2) continuity: if $\left\|X_{i}-X\right\|_{* \infty} \rightarrow 0$, then $\mathbb{U}\left(X_{i}\right) \rightarrow$ $\mathbb{U}(X)$.

Then we have the following nonlinear expected utility theorem which generalized the well-known von NeumanMorgenstern's axiom on expected utility. 
Proposition 8. Let $\mathbb{E}[\cdot]$ be a strictly monotonic expectation satisfying (i) and (ii) in Definition 1 . One assumes that $\mathbb{E}[\cdot]$ is continuous in $\mathscr{H}$ and let $u(\cdot)$ be a continuous and strictly increasing function $u(\cdot): R \rightarrow R$. Then the functional $U(\cdot)$ defined by

$$
U(X):=\mathbb{E}[u(X)]
$$

is a utility functional satisfying (u1) and (u2).

Conversely, for each given utility $U(\cdot)$ satisfying (u1) and $(\mathrm{u} 2)$, there exist a strict monotonic nonlinear expectation $\mathbb{E}[\cdot]$ and a continuous and strictly increasing function $u(\cdot): R \rightarrow$ $R$ such that (15) holds.

\section{Stochastic Dominance under the Nonlinear Expected Utilities}

Using nonlinear expected utility to determine the advantages between two random choices is only for a single economic actor. Here comes a problem: can we raise the same question to a group of economic actors? If we still discuss it by using nonlinear expected utility, this means asking the same question to a class of expected utility functions.

In mathematics, it can form such a problem: suppose $\mathscr{H}$ is a collection of random variables. $\mathcal{U}$ is a class of strictly increasing and continuously differentiable functions, which represents the collection of all the utility functions of an investor group. Define a partial ordering $\succeq$ in $\mathscr{H}$ : for any $X, Y \in \mathscr{H}$,

$$
X \geq Y \Longleftrightarrow \forall u \in \mathcal{U}, \quad \mathbb{E}[u(X)] \geq \mathbb{E}[u(Y)] .
$$

Here $\mathbb{E}[\cdot]$ is nonlinear expectation, $X, Y$ can be regarded as two risky securities, and $u \in \mathcal{U}$ can be an investor's expected utility function. Thus, this definition of the partial ordering means that all members of the investor group think the former is better than the latter. Here it is important to note that, in general, this is a partially ordering rather than a complete ordering. That is to say, for some pairs of risky securities, neither one stochastically dominates the other, and yet they cannot be said to be equal. At the same time, it is just investor group's preferences characterized by the expected utilities functions theory.

Definition 9. The above-mentioned partial ordering is called stochastic dominance under the nonlinear expected utility.

Remark 10. Stochastic dominance is a form of stochastic ordering. The term is used in decision theory and decision analysis to refer to situations where one random choice can be ranked as superior to another. It is based on preferences regarding outcomes. In linear expected utility, there are first-order stochastic dominance and second-order stochastic dominance and so on. For more results, see [13-16].

Definition 11. In Definition 9, if for any $X, Y \in \mathscr{H}$,

$$
X>Y \Longleftrightarrow \forall u \in \mathcal{U}, \quad \mathbb{E}[u(X)]>\mathbb{E}[u(Y)],
$$

then the partial ordering is called strictly stochastic dominance under the nonlinear expected utility.
Next, we give the main results of this paper.

Theorem 12. Let $(\Omega, \mathscr{H}, \mathbb{E})$ be a nonlinear expectation space, $X, Y \in \mathscr{H}$, and $\mathcal{U}$ a class of strictly increasing and continuously differentiable functions. If any of the following conditions is satisfied:

(1) $X \geq Y$,

(2) the distribution of $X$ is stronger than $Y$, namely,

$$
\mathbb{E}[\varphi(X)] \geq \mathbb{E}[\varphi(Y)], \quad \text { for each } \varphi \in \mathscr{C}_{l, \text { lip }}(R),
$$

then $X$ stochastically dominates $Y$, that is,

$$
\mathbb{E}[u(X)] \geq \mathbb{E}[u(Y)], \quad \forall u \in \mathcal{U} .
$$

Proof. (1) If $X \geq Y$, then

$$
\forall u \in \mathcal{U}, \quad \mathbb{E}[u(X)] \geq \mathbb{E}[u(Y)]
$$

is easily concluded by the fact that $u \in \mathcal{U}$ is strictly increasing and the monotonicity of $\mathbb{E}[\cdot]$.

(2) First, notice that an everywhere differentiable function $g(\cdot)$ which is a lipschitz continuous with $k=\sup \left|g^{\prime}(x)\right|$ is equivalent to the fact that $g(\cdot)$ has bounded first derivative. In particular, any continuously differentiable function is locally lipschitz, as continuous functions are locally bounded so its gradient is locally bounded as well. It means that for all $u \in$ $\mathcal{U}, u \in \mathscr{C}_{l \text { lip }}(R)$. So if the distribution of $X$ is stronger than that of $Y$, we have

$$
\mathbb{E}[\varphi(X)] \geq \mathbb{E}[\varphi(Y)], \quad \text { for each } \varphi \in \mathscr{C}_{l, \text { lip }}(R) .
$$

Because for all $u \in \mathscr{U}, u \in \mathscr{C}_{l \text { lip }}(R)$; then we can have

$$
\forall u \in \mathcal{U}, \quad \mathbb{E}[u(X)] \geq \mathbb{E}[u(Y)] .
$$

So the result holds by Definition 9.

Remark 13. ( $\alpha$ ) The above conclusion (2) gives sufficient condition on which a random choice $X$ stochastically dominates a random choice $Y$ under the nonlinear expected utilities. It is a general extension of the first-order stochastic dominance under the linear expectation utility.

$(\beta)$ The above conclusion (1) is very intuitive. Next, we give an example which is not intuitive.

Example 14. Suppose

$$
\Omega=\left\{\omega_{1}, \omega_{2}\right\},
$$

and we have two probabilities $\{2 / 3,1 / 3\}$ and $\{2 / 5,3 / 5\}$ denoted by $\mathbb{P}$ and $\mathbb{Q}$, respectively, where

$$
\begin{aligned}
& \mathbb{P}\left(\left\{\omega_{1}\right\}\right)=\frac{2}{3}, \\
& \mathbb{P}\left(\left\{\omega_{2}\right\}\right)=\frac{1}{3}, \\
& \mathbb{Q}\left(\left\{\omega_{1}\right\}\right)=\frac{2}{5}, \\
& \mathbb{Q}\left(\left\{\omega_{2}\right\}\right)=\frac{3}{5} .
\end{aligned}
$$


We assume $\mathscr{H}$ is a collection of random variables, and $\mathcal{U}$ is a class of strictly increasing and continuously differentiable functions. Take the nonlinear expectation utility like the following:

$$
\begin{array}{r}
\mathbb{E}[u(\xi)]=\frac{3}{4} \max \left\{E_{\mathbb{P}}[u(\xi)], E_{\mathbb{Q}}[u(\xi)]\right\} \\
+\frac{1}{4} \min \left\{E_{\mathbb{P}}[u(\xi)], E_{\mathbb{Q}}[u(\xi)]\right\}, \\
u \in \mathcal{U}, \quad \xi \in \mathscr{H} .
\end{array}
$$

We set

$$
\begin{aligned}
& X\left(\omega_{1}\right)=1, \\
& X\left(\omega_{2}\right)=0, \\
& Y\left(\omega_{1}\right)=0, \\
& Y\left(\omega_{2}\right)=1 .
\end{aligned}
$$

For all $u \in \mathscr{U}$, we can calculate the following results:

$$
\begin{aligned}
& E_{\mathbb{P}}[u(X)]=\frac{2}{3} u(1)+\frac{1}{3} u(0), \\
& E_{\mathbb{Q}}[u(X)]=\frac{2}{5} u(1)+\frac{3}{5} u(0), \\
& \mathbb{E}[u(X)]=\frac{6}{15} u(0)+\frac{9}{15} u(1), \\
& E_{\mathbb{P}}[u(Y)]=\frac{2}{3} u(0)+\frac{1}{3} u(1), \\
& E_{\mathbb{Q}}[u(X)]=\frac{2}{5} u(0)+\frac{3}{5} u(1), \\
& \mathbb{E}[u(Y)]=\frac{7}{15} u(0)+\frac{8}{15} u(1),
\end{aligned}
$$

Since $u \in \mathscr{U}$ is strictly increasing, then $u(1)>u(0)$; so

$$
\begin{aligned}
\mathbb{E}[u(X)]-\mathbb{E}[u(Y)]=\frac{1}{15} u(1)-\frac{1}{15} u(0)>0, & u \in \mathcal{U} . \\
& \\
\mathbb{E}[u(X)]>\mathbb{E}[u(Y)], \quad u \in \mathcal{U} . &
\end{aligned}
$$

Hence we can say that $X$ strictly stochastic dominates $Y$.

It is easy to see that neither $X \geq Y$ nor $Y \geq X$ in a whole. We can check that $X$ strictly stochastic dominates $Y$. This is not the intuitive way; this implies that stochastic dominance by the nonlinear expected utilities is meaningful.

When $\mathbb{E}[\cdot]$ is a sublinear expectation, Theorem 12 is still valid. Furthermore, we can also have the following theorem.

Theorem 15. Let $(\Omega, \mathscr{H}, \mathbb{E})$ be a sublinear expectation space, $X, Y \in \mathscr{H}$, and $\mathcal{U}$ a class of strictly increasing and continuously differentiable functions. If $X \geq Y$ q.s., then $X$ stochastically dominates $Y$, that is,

$$
\mathbb{E}[u(X)] \geq \mathbb{E}[u(Y)], \quad \forall u \in \mathcal{U} .
$$

Proof. We claim that if $X \geq Y$ q.s., then $\mathbb{E}[X] \geq \mathbb{E}[Y]$.

This is because we can get $\mathbb{V}(X<Y)=0$ by $X \geq Y$ q.s., which means $\mathbb{V}(X<Y)=0, v(X \geq Y)=1$; namely,

$$
v(X \geq Y)=\inf _{\mathbb{P} \in \mathscr{P}} \mathbb{P}(X \geq Y)=1
$$

Then we can get

$$
\forall \mathbb{P} \in \mathscr{P}, \quad E_{\mathbb{P}}[X] \geq E_{\mathbb{P}}[Y]
$$

so

$$
\sup _{\mathbb{P} \in \mathscr{P}} \mathbb{E}_{\mathbb{P}}(X) \geq \sup _{\mathbb{P} \in \mathscr{P}} \mathbb{E}_{\mathbb{P}}(Y)
$$

According to the represent theorem, we have $\mathbb{E}[X] \geq \mathbb{E}[Y]$.

Since $X \geq Y$ q.s. and $u \in \mathcal{U}$ is strictly increasing, $u(X) \geq$ $u(Y)$ q.s. is available by the same procedure as above. So we can obtain

$$
\mathbb{E}[u(X)] \geq \mathbb{E}[u(Y)], \quad \forall u \in \mathscr{U}
$$

by the above conclusion.

Next, we shall give a lemma first, then present a strictly stochastic dominance result under sublinear expectations.

Lemma 16. Let $(\Omega, \mathscr{H}, \mathbb{E})$ be a sublinear expectation space, $Y \in \mathscr{H}$, and $\mathcal{U}$ a class of strictly increasing and continuously differentiable functions. If $u(Y)$ has no mean uncertainty, that is,

$$
\mathbb{E}[u(Y)]=-\mathbb{E}[-u(Y)],
$$

then

$$
\mathbb{E}[u(Y)]=E_{\mathbb{P}}[u(Y)], \quad \forall \mathbb{P} \in \mathscr{P} .
$$

Proof. Since

$$
\begin{gathered}
\mathbb{E}[u(Y)]=\sup _{\mathbb{P} \in \mathscr{P}} \mathbb{E}_{\mathbb{P}}[u(Y)], \\
-\mathbb{E}[-u(Y)]=-\sup _{\mathbb{P} \in \mathscr{P}} \mathbb{E}_{\mathbb{P}}[-u(Y)]=-\sup _{\mathbb{P} \in \mathscr{P}}\left(-\mathbb{E}_{\mathbb{P}}[u(Y)]\right) \\
=-\left(-\inf _{\mathbb{P} \in \mathscr{P}}\left(\mathbb{E}_{\mathbb{P}}[u(Y)]\right)\right)=\inf _{\mathbb{P} \in \mathscr{P}}\left(\mathbb{E}_{\mathbb{P}}[u(Y)]\right),
\end{gathered}
$$

we can get

$$
\sup _{\mathbb{P} \in \mathscr{P}} \mathbb{E}_{\mathbb{P}}[u(Y)]=\inf _{\mathbb{P} \in \mathscr{P}}\left(\mathbb{E}_{\mathbb{P}}[u(Y)]\right) .
$$

Then

$$
\begin{aligned}
\mathbb{E}[u(Y)] & =\sup _{\mathbb{P} \in \mathscr{P}} \mathbb{E}_{\mathbb{P}}[u(Y)]=\inf _{\mathbb{P} \in \mathscr{P}}\left(\mathbb{E}_{\mathbb{P}}[u(Y)]\right) \\
& =E_{\mathbb{P}}[u(Y)], \quad \forall \mathbb{P} \in \mathscr{P} .
\end{aligned}
$$


Theorem 17. Let $(\Omega, \mathscr{H}, \mathbb{E})$ be a sublinear expectation space, $X, Y \in \mathscr{H}$, and $\mathcal{U}$ a class of strictly increasing and continuously differentiable functions. If $X \geq Y$ q.s., $\mathbb{V}(X>Y)>0$, and $\mathbb{E}[u(Y)]=-\mathbb{E}[-u(Y)]$, for all $u \in \mathcal{U}$, that is, $u(Y)$ has no mean uncertainty, then $X$ strictly stochastically dominates $Y$, that is,

$$
\mathbb{E}[u(X)]>\mathbb{E}[u(Y)], \quad \forall u \in \mathcal{U}
$$

Proof. Since $X \geq Y$ q.s. and $\mathbb{V}(X>Y)>0$, there exists $\mathbb{P} \in$ $\mathscr{P}$, such that

$$
\begin{aligned}
& \mathbb{P}(X \geq Y)=1, \\
& \mathbb{P}(X>Y)>0 .
\end{aligned}
$$

Therefore

$$
E_{\mathbb{P}}[X]>E_{\mathbb{P}}[Y] .
$$

Since

$$
\mathbb{E}[u(Y)]=-\mathbb{E}[-u(Y)], \quad \forall u \in \mathcal{U},
$$

according to Lemma 16, we have

$\mathbb{E}[u(X)] \geq E_{\mathbb{P}}[u(X)]>E_{\mathbb{P}}[u(Y)]=\mathbb{E}[u(Y)], \quad \forall u \in \mathcal{U}$.

Corollary 18. Let $(\Omega, \mathscr{H}, \mathbb{E})$ be a sublinear expectation space, $X, Y \in \mathscr{H}$, and $X \geq Y$ q.s. Assume $u(x)=k x, k>0$. If $\mathbb{V}(X>Y)>0$ and $Y$ has no mean uncertainty, that is, $\mathbb{E}[Y]=$ $-\mathbb{E}[-Y]$, then

$$
\mathbb{E}[u(X)]>\mathbb{E}[u(Y)] .
$$

Proof. If $u(x)=k x, k>0$, and $Y$ has mean certainty, it is easy to verify that $u(Y)$ has mean certainty; that is, $\mathbb{E}[u(Y)]=$ $-\mathbb{E}[-u(Y)]$. Then the consequence attains immediately by Theorem 17.

Remark 19. This corollary gives sufficient condition for the result that $X$ strictly stochastically dominates $Y$ to the riskneutral group.

Corollary 20. Let $(\Omega, \mathscr{H}, \mathbb{E})$ be a sublinear expectation space, $X, Y \in \mathscr{H}$, and $\mathcal{U}$ a class of strictly increasing and continuously differentiable functions. If $X \geq Y$ q.s., $v(X \leq Y)<1$, and $\mathbb{E}[u(Y)]=-\mathbb{E}[-u(Y)]$, for all $u \in \mathcal{U}$, that is, $u(Y)$ has no mean uncertainty, then $X$ strictly stochastically dominates $Y$, that is,

$$
\mathbb{E}[u(X)]>\mathbb{E}[u(Y)], \quad \forall u \in \mathcal{U}
$$

Proof. By using the relationship between $\mathbb{V}$ and $v$, the consequence attains immediately by Theorem 17.

Next, we give an example to apply Theorem 17.
Example 21. Suppose there is an outcome $\Omega=[0,1]$, which indicates the market conditions. $\mathscr{F}$ is the $\sigma$-algebra of Borel sets on $\Omega$ and $|\cdot|$ is the Lebesgue measure on $[0,1]$.

We have two prior probabilities denoted by $\mathbb{P}$ and $\mathbb{Q}$, respectively, where

$$
\begin{array}{r}
\mathbb{P}(A)=\frac{1}{2}\left|A \cap\left[0, \frac{1}{3}\right)\right|+\left|A \cap\left[\frac{1}{3}, \frac{2}{3}\right)\right|+\frac{3}{2}\left|A \cap\left[\frac{2}{3}, 1\right]\right|, \\
A \in \mathscr{F}, \\
\mathbb{Q}(A)=\frac{1}{4}\left|A \cap\left[0, \frac{1}{3}\right)\right|+\frac{5}{4}\left|A \cap\left[\frac{1}{3}, \frac{2}{3}\right)\right|+\frac{3}{2}\left|A \cap\left[\frac{2}{3}, 1\right]\right|, \\
A \in \mathscr{F} .
\end{array}
$$

We assume $\mathscr{H}$ is a collection of random variables, which represents risky securities and $\mathcal{U}$ is a class of strictly increasing and continuously differentiable functions, which represents the collection of all the utility functions of an investor group. Take the sublinear expectation utility as follows:

$$
\begin{array}{r}
\mathbb{E}[u(\xi)]=\max \left\{E_{\mathbb{P}}[u(\xi)], E_{\mathbb{Q}}[u(\xi)]\right\}, \\
u \in \mathscr{U}, \quad \xi \in \mathscr{H} .
\end{array}
$$

There are two risky securities

$$
\begin{aligned}
& X(\omega)= \begin{cases}-10, & \omega=0, \\
0, & \omega \in\left(0, \frac{1}{3}\right), \\
1, & \omega \in\left[\frac{1}{3}, \frac{2}{3}\right), \\
10, & \omega \in\left[\frac{2}{3}, 1\right],\end{cases} \\
& Y(\omega)= \begin{cases}0, & \omega \in\left[0, \frac{2}{3}\right) \\
10, & \omega \in\left[\frac{2}{3}, 1\right] .\end{cases}
\end{aligned}
$$

It is clear that above conditions guarantee Theorem 17; therefore we have that $X$ strictly stochastically dominates $Y$, that is,

$$
\mathbb{E}[u(X)]>\mathbb{E}[u(Y)], \quad u \in \mathcal{U}
$$

This means that all members of the investor group think the former is better than the latter.

In fact, for all $u \in \mathcal{U}$, we can calculate the following results:

$$
\begin{gathered}
E_{\mathbb{P}}[u(X)]=\frac{1}{6} u(0)+\frac{1}{3} u(1)+\frac{1}{2} u(10) \\
E_{\mathbb{Q}}[u(X)]=\frac{1}{12} u(0)+\frac{5}{12} u(1)+\frac{1}{2} u(10)
\end{gathered}
$$


then

$$
\begin{gathered}
\mathbb{E}[u(X)]=\frac{1}{12} u(0)+\frac{5}{12} u(1)+\frac{1}{2} u(10) ; \\
E_{\mathbb{P}}[u(Y)]=\frac{1}{2} u(0)+\frac{1}{2} u(10) \\
E_{\mathbb{Q}}[u(Y)]=\frac{1}{2} u(0)+\frac{1}{2} u(10) ;
\end{gathered}
$$

then

$$
\mathbb{E}[u(Y)]=\frac{1}{2} u(0)+\frac{1}{2} u(10) .
$$

Since $u \in \mathcal{U}$ is strictly increasing, then $u(1)>u(0)$; so

$$
\begin{aligned}
\mathbb{E}[u(X)]-\mathbb{E}[u(Y)]=\frac{5}{12}(u(1)-u(0)) & >0, \\
u & \in \mathcal{U} .
\end{aligned}
$$

Then

$$
\mathbb{E}[u(X)]>\mathbb{E}[u(Y)], \quad u \in \mathcal{U}
$$

that is, $X$ strictly stochastically dominates $Y$.

\section{Conclusion}

In this paper, we study stochastic dominance under the nonlinear expected utilities. First we attain sufficient conditions on which a random choice $X$ stochastically dominates a random choice $Y$ under the nonlinear expected utilities; then we attain sufficient conditions on which strictly stochastic dominance of a random choice $X$ over a random choice $Y$ under the sublinear expected utilities; finally we give sufficient condition for strictly stochastic dominance of $X$ over $Y$ under the sublinear expected utilities to the riskneutral group.

\section{Conflict of Interests}

The author declares that there is no conflict of interests regarding the publication of this paper.

\section{Acknowledgments}

Xiao's researches are supported by the NNSF project (11301309) of China, NSF project (ZR2011AQ007) of Shandong Province of China, Project of Shandong Province Higher Educational Science and Technology Program (J11LA08), Project of Shandong Provincial Scientific Research Foundation for Excellent Young Scientists (BS2013SF003), and the Postdoctoral Science Foundation of China (2013M540540).

\section{References}

[1] J. von Neumann and O. Morgenstern, Theory of Games and Economic Behavior, Princeton University Press, Princeton, NJ, USA, 1953.
[2] M. Allais, "La paychologie de I'home rationnel devant le risqu: critique des postulats et axioms del'cole Americaine," Econometrica, vol. 21, no. 1953, pp. 503-546, 1979, translated and reprinted in Allais and Hagen.

[3] S. Peng, "Nonlinear expectations and stochastic calculus under uncertainty," http://arxiv.org/abs/1002.4546.

[4] X. Li, "On the strict comparison theorem for G-expectations," http://arxiv.org/abs/1002.1765.

[5] M. Hu, S. Ji, S. Peng, and Y. Song, "Comparison theorem, Feynman-Kac formula and Girsanov transformation for BSDEs driven by G-Brownian motion," Stochastic Processes and Their Applications, vol. 124, no. 2, pp. 1170-1195, 2014.

[6] M. Hu, S. Ji, S. Peng, and Y. Song, "Backward stochastic differential equations driven by G-Brownian motion," Stochastic Processes and Their Applications, vol. 124, no. 1, pp. 759-784, 2014.

[7] M. Hu, S. Ji, and S. Yang, "A stochastic recursive optimal control problem under the G-expectation framework," Applied Mathematics \& Optimization, 2014.

[8] S. Peng, "G-Brownian motion and dynamic risk measure under volatility uncertainty," http://arxiv.org/abs/0711.2834.

[9] L. G. Epstein and S. Ji, "Ambiguous volatility, possibility and utility in continuous time," Journal of Mathematical Economics, vol. 50, pp. 269-282, 2014.

[10] L. G. Epstein and S. Ji, "Ambiguous volatility and asset pricing in continuous time," Review of Financial Studies, vol. 26, no. 7, pp. 1740-1786, 2013.

[11] X. Cao, "An upper bound of large deviations for capacities," Mathematical Problems in Engineering, vol. 2014, Article ID 516291, 6 pages, 2014.

[12] S. Peng, "Nonlinear expectations and nonlinear Markov chains," Chinese Annals of Mathematics B, vol. 26, no. 2, pp. 159-184, 2005.

[13] V. S. Bawa, "Optimal rules for ordering uncertain prospects," Journal of Financial Economics, vol. 2, no. 1, pp. 95-121, 1975.

[14] D. Dentcheva and A. Ruszczyski, "Optimization with stochastic dominance constraints," SIAM Journal on Optimization, vol. 14, no. 2, pp. 548-566, 2003.

[15] D. Dentcheva and A. Ruszczyński, "Semi-infinite probabilistic optimization: first-order stochastic dominance constraints," Optimization, vol. 53, no. 5-6, pp. 583-601, 2004.

[16] E. Jonathan and Jr. Ingersoll, Theory of Financial Decision Making, Rowman and Little field Publishers, 1987. 


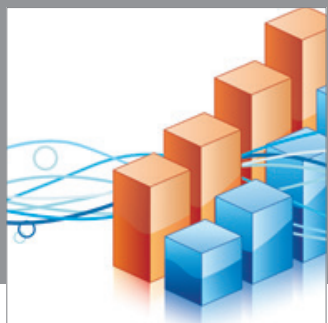

Advances in

Operations Research

mansans

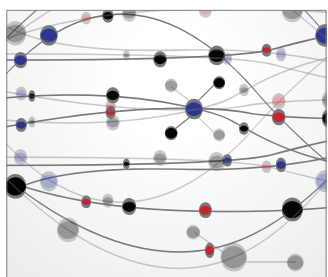

The Scientific World Journal
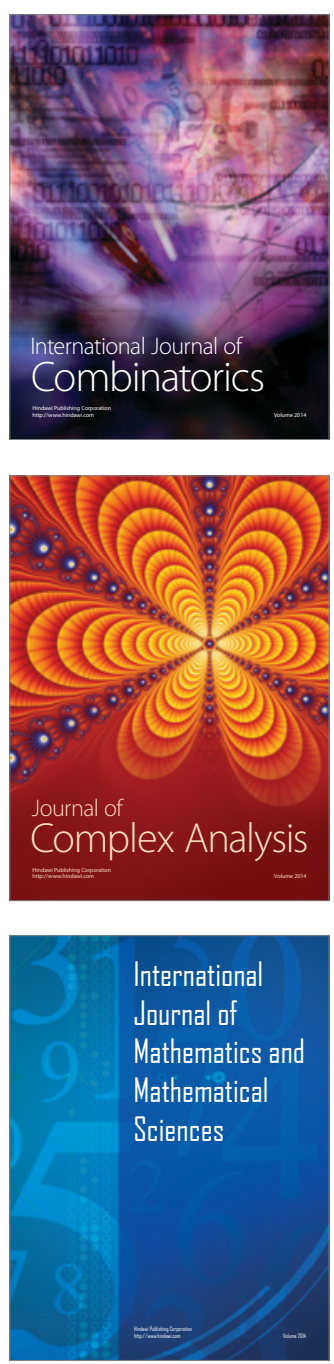
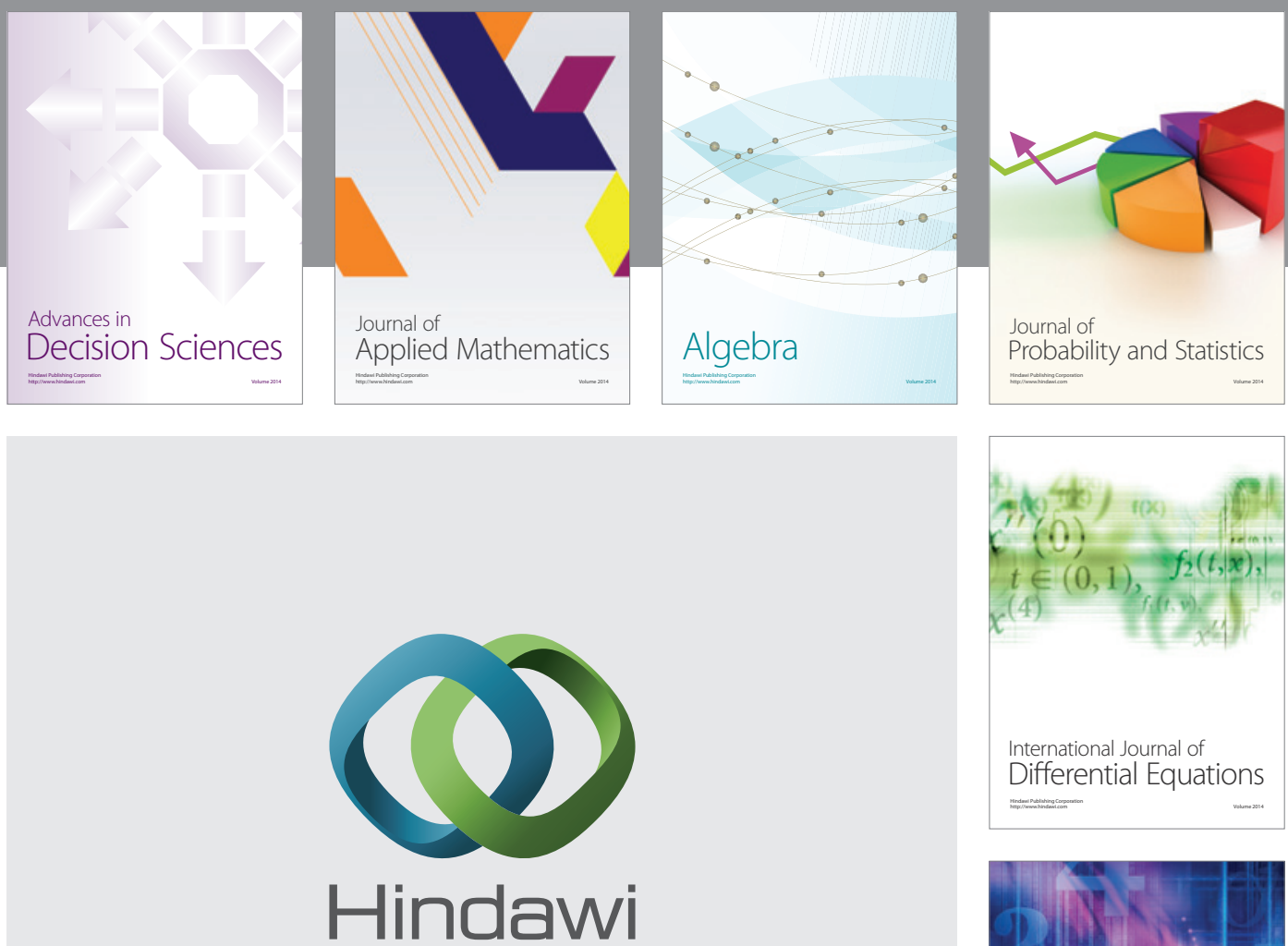

Submit your manuscripts at http://www.hindawi.com
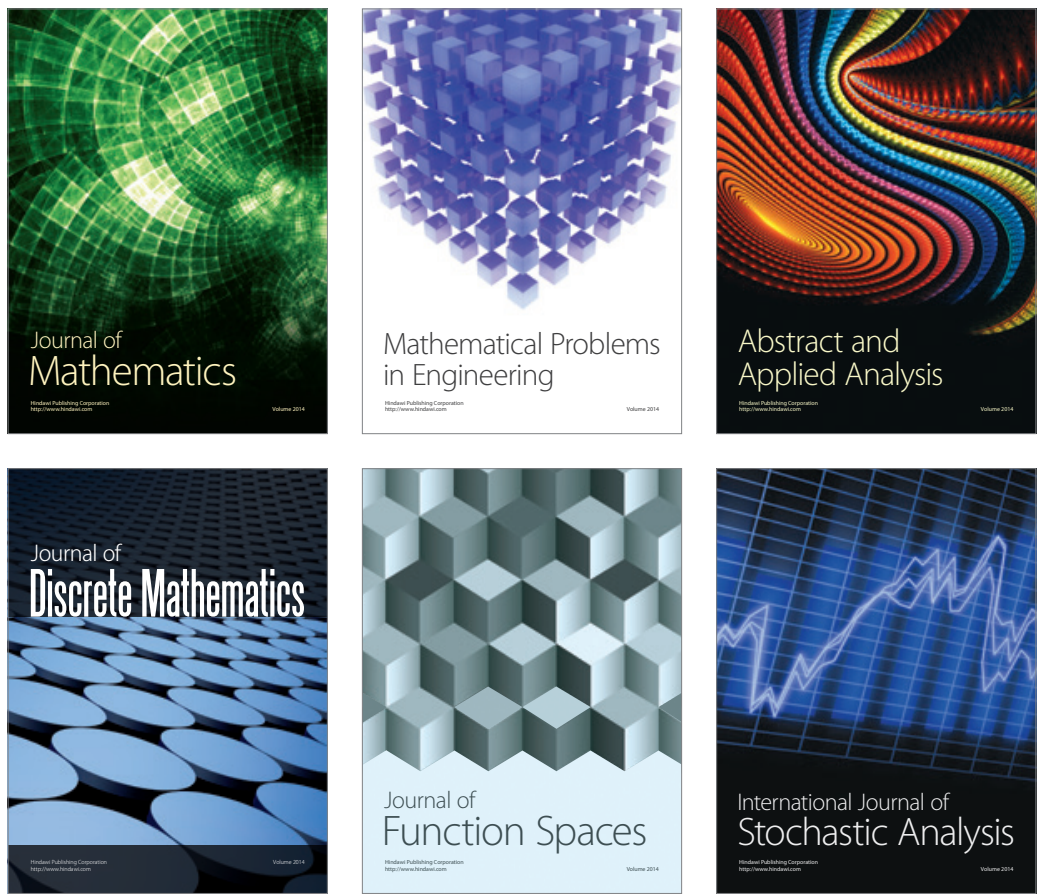

Journal of

Function Spaces

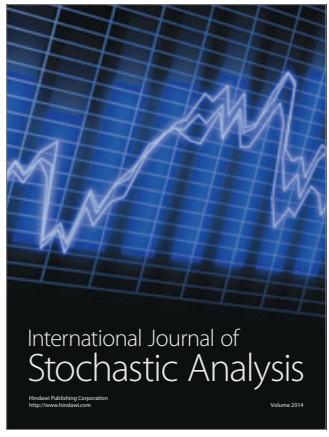

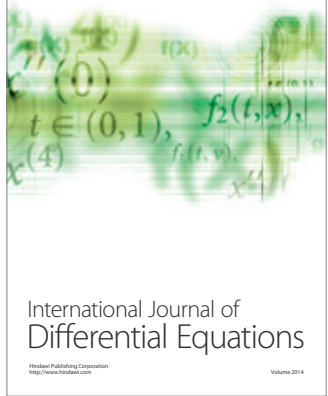
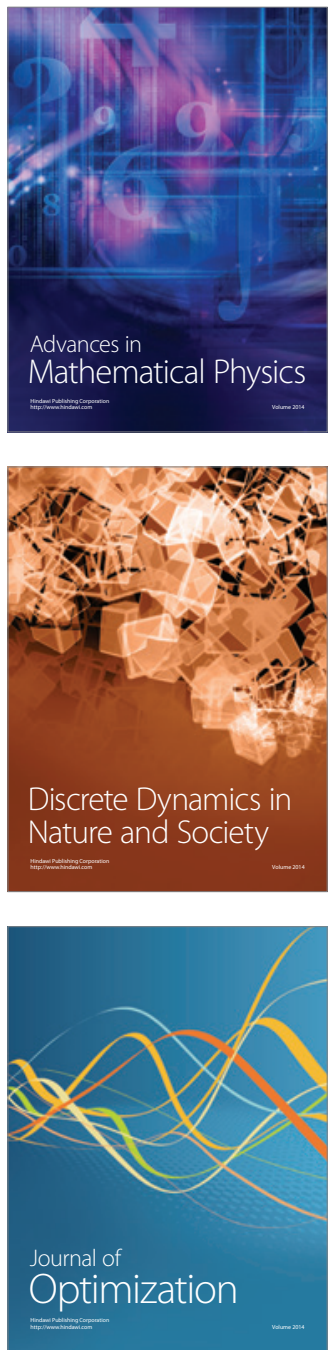